\title{
Performance Analysis of OLSR Protocol for Wireless Sensor Networks and Comparison Evaluation with AODV Protocol
}

\author{
Tao Yang $\nmid$, Leonard Barolli†, Makoto Ikeda $\dagger$, Fatos Xhafa $\dagger \dagger$, Arjan Durresi $\dagger$ \\ †Graduate School of Engineering \\ Fukuoka Institute of Technology (FIT) \\ 3-30-1 Wajiro-Higashi, Higashi-Ku, Fukuoka 811-0295, Japan \\ E-mail: $\{$ bd07003, bd07001\}@bene.fit.ac.jp \\ $\ddagger$ Department of Information and Communication Engineering \\ Fukuoka Institute of Technology (FIT) \\ 3-30-1 Wajiro-Higashi, Higashi-Ku, Fukuoka 811-0295, Japan \\ E-mail: barolli@fit.ac.jp \\ $\dagger \dagger$ Department of Languages and Informatics Systems \\ Technical University of Catalonia \\ C/Jordi Girona 1-3, 08034 Barcelona, Spain \\ E-mail: fatos@1si.upc.edu \\ $\dagger \ddagger$ Department of Computer and Information Science \\ Indiana University Purdue University at Indianapolis (IUPUI) \\ 723 W. Michigan Street SL 280 \\ Indianapolis, IN 46202, USA \\ E-mail: durresi@cs.iupui.edu
}

\begin{abstract}
Sensor networks are a sensing, computing and communication infrastructure that are able to observe and respond to phenomena in the natural environment and in our physical and cyber infrastructure. The sensors themselves can range from small passive microsensors to larger scale, controllable weather-sensing platforms. Presently, there are many research work for sensor networks. In our previous work, we implemented a simulation system for sensor networks and simulated the proposed system with reactive and preactive protocols. In this work, we want to investigate how the sensor network performs in case of using OLSR protocol and compare the simulation results with AODV protocol. The simulation results have shown that the consumed energy for OLSR protocol is better than AODV protocol. Also, the goodput for the case of using OLSR does not change too much compared with the case using AODV, but the goodput is not good when the number of nodes is increased.
\end{abstract}

\section{Introduction}

Wireless Sensor Network (WSN) is traditionally composed of devices (in the following named also nodes) distributed over a monitored region, which generate samples of a given phenomenon (e.g. atmospheric pressure, or temperature measurements) and forward them to a sink that collects the information possibly through a muti-hop wireless network. Recently, we witnessed a lot of research effort towards the optimization of standard communication paradigms for such networks. In fact, the traditional Wireless Network (WN) design has never paid attention to constraints such as the limited or scarce energy of nodes and their computational power. Another aspect which is different from traditional WN is the communication reliability and congestion control. In traditional wired nets, one reasonably supposes that communication paths are stable along the transmission instances. This fact permits to use the end-to-end approach to the design of reliable transport and application protocols. The TCP works well because of the stability of links. On the other hand, in WSN paths can 


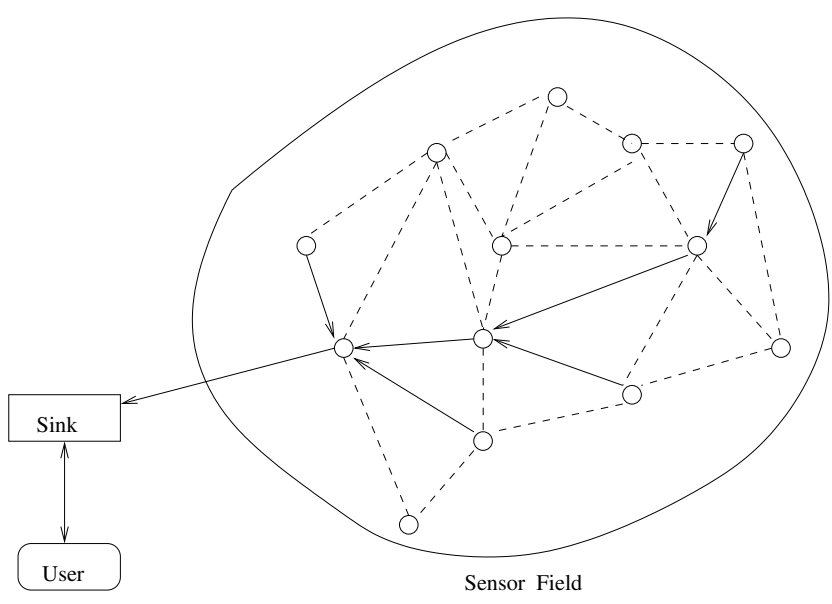

Figure 1. Physical architecture of WSN.

change over time, because of time-varying characteristics of links and nodes reliability. These problems are important especially in a multi-hop scenario, where nodes accomplish also at the routing of other nodes' packets.

Recently, there are many research work for sensor networks [1, 2, 3, 4]. In our previous work [5], we implemented a simulation system for sensor networks. We carried out simulations for lattice and random topology with TwoRayGround and Shadowing radio models considering three protocols: Ad-hoc On-demand Distance Vector (AODV), Dynamic Source Routing (DSR) and DestinationSequenced Distance Vector (DSDV). In this paper, we compare the simulation results for two protocols: AODV and OLSR. The simulation results have shown that the consumed energy for OLSR protocol is better than AODV protocol.Also, the goodput for the case of using OLSR does not change too much compared with the case using AODV, but the goodput is not good when the number of nodes is increased.

The remainder of the paper is organized as follows. In Section 2, we explain the proposed network simulation model. In Section 3, we discuss the goodput and depletion concepts. In Section 4, we show the simulation results. Conclusions of the paper are given in Section 5.

\section{Proposed Network Simulation Model}

In our WSNs, every node detects the physical phenomenon and sends back to the sink node data packets. In Fig. 1 is shown the physical architecture of WSN. We suppose that the sink node is more powerful that sensor nodes and it is always located at the borders of the service area. We analyze the performance of the network in a fixed time interval. This is the available time for the detection of the phenomenon and its value is application dependent. Proposed network simulation model is shown in Fig.2.

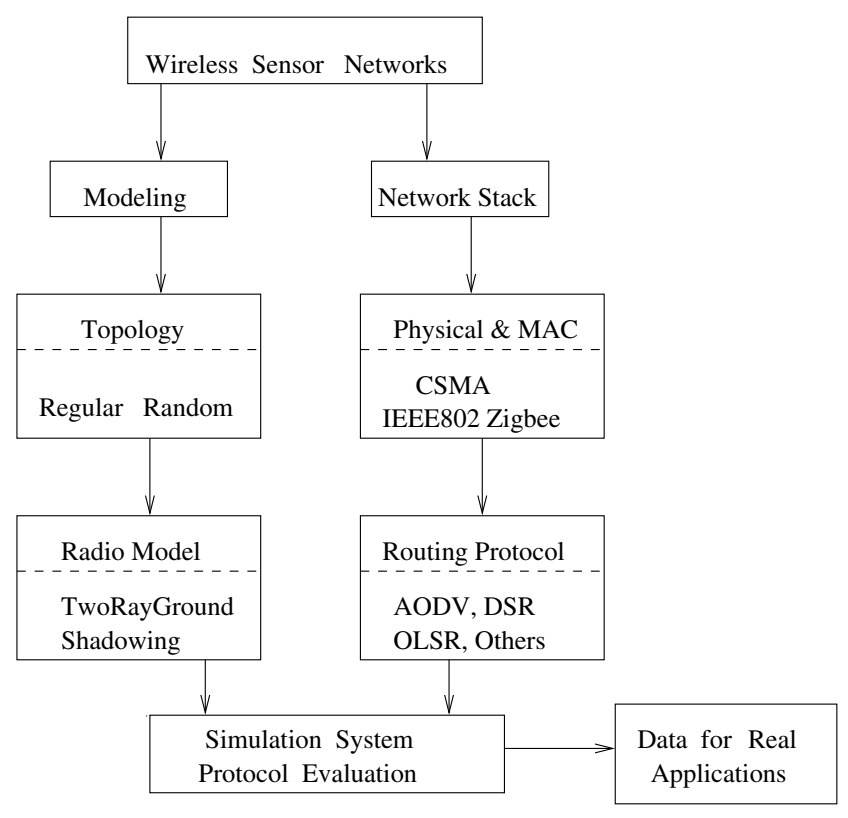

Figure 2. Network simulation model.

In this paper, we perform the simulations for TwoRayGround radio model and lattice topology and compare the goodput and depletion of OLSR and AODV protocols.

\subsection{Routing Protocols}

We are aware of many proposals of routing protocols for ad-hoc networks. Here, we consider proactive protocol OSLR [6] and reactive protocol AODV.

\subsubsection{OLSR}

OLSR is a proactive routing protocol, providing advantage of having routes immediately available in each node for all destinations in the network. It is an optimization of a pure Link State routing protocol. This optimization is based on the concept of MultiPoint Relays (MPRs). First, using multipoint relays reduces the size of the control message: rather than declaring all link, a node declares only the set of links with its neighbors that are its "multipoint relays". The use of MPRs also minimizes flooding of control traffic. Indeed only MPRs forward control messages. This technique significantly reduces the number of retransmissions of broadcast control messages. OLSR is characterized by two types of control messages: neighborhood and topology messages, called respectively Hello messages and Topology Control (TC) messages. OLSR provides two main functionalities: the first is Neighbor Discovery, and the second is Topology Dissemination. These will be described in following. 


\section{Neighbor Discovery}

Each node must detect the neighbor nodes with which it has a direct link. Due to the uncertainties in radio propagation, a link between neighboring nodes may enable the transmission of data in either one or both directions over the link. For this, each node periodically broadcasts Hello messages, containing the list of neighbors known to each node and their link status. The link status can be either symmetric (if communication is possible in both directions), asymmetric (if communication is only possible in one direction), multipoint relay (if the link is symmetric and the sender node of the Hello message has selected this node as a MPR), or lost (if the link has been lost). The Hello messages are received by all one-hop neighbors, but are not forwarded. They are broadcasted at low frequency determined by refreshing priod of "HELLO-INTERVAL". Thus, Hello messages enable each onde to discover its one-hop neighbors, as well as its two-hop neighbors. This neighborhood and two-hop neighborhood information has an associated holding time "NEIGHBOR-HOLD-TIME", after which it is no longer valid.

On the basis of this information, each node $m$ of the network independently selects its own set of MPRs among its one-hop neighbors in such a way that all two-hop neighbors of $m$ have symetric link with $\operatorname{MPR}(\mathrm{m})$. This means that the MPRs cover (in terms of radio range) all the two-hop neighbors. Fig. 3 shows the MPRs seletion by node $m$. A possible algorithm to selet these MPRs is described in [7]. The MPR set is computed whenever a change in the onehop neighborhood or two-hop neighborhood is detected. In addition, each node $m$ maintains the set of its "MPR selectors". This set contains the nodes which have selected $m$ as a MPR. Node $m$ only forwards broadcast messages received from one of its MPR selectors.

\section{Topology Dissemination}

Each node of the network maintains topological information about the network obtained by means of TC messages. Each node $m$ selected as a MPR, broadcasts a TC message at least every "TC-INTERVAL". If a change occours in the MPR selector set, the next TC can be sent earlier (e.g. after some pre-specified minimum interval). The TC messages are flooded to all nodes in the network and take advantage of MPRs to reduce the number of retransmissions. The TC message originated from node $m$ declares the MPR selectors of $m$. Thus, a node is reachable either directly or via its MPRs. This topological information collected in each node has an associated holding time "TOP-HOLD-TIME", after which it is no longer valid.

The neighbor information and the topology information are refreshed periodically, and they enable each node to compute the routes to all known destinations. These routes are computed with Dijkstra's shortest path algorithm. Hence, they are optimal concerning the number of hops. Moreover, for any route, any intermediate node on this route is a MPR of the next node. The routing table is computed whenever there is a change in neighborhood information or a change in topology information.

\section{OLSR "gateways"}

Each node maintains information concerning which nodes may act as "gateways" to associated hosts and networks. Those "gateways" generate periodically a "HNA" (Host and Network Association) message, containing pairs of (network address, netmask) corresponding to the connected hosts and networks. The "HNA" messages are flooded to all the nodes in the network by the MPRs. Those messsages should be transmitted periodically every "HNAINTERVAL". The collected information is valid for "HNAHOLD-TIME". The networks and associated hosts are added to the routing table and they have the same next hop as the one to reach the appropriate "gateways".

\section{Multiple Interface Declaration}

In the full OLSR protocol, a node which has several interfaces, periodically emits a special type message, "Multiple Interface Declaration", in which it lists all its interfaces address, along with one of them, fix, which it (arbitrarily) chooses as its main address.

\subsubsection{AODV}

The AODV is an improvement of DSDV to on-demand scheme. It minimize the broadcast packet by creating route only when needed. Every node in network should maintain route information table and participate in routing table exchange. When source node wants to send data to the destination node, it first initiates route discovery process. In this process, source node broadcasts Route Request (RREQ) packet to its neighbors. Neighbor nodes which receive RREQ forward the packet to its neighbor nodes. Neighbor nodes which receive RREQ forward the packet to its neighbors, and so on. This process continues until RREQ reach to the destination or the node who know the path to destination. When the intermediate nodes receive RREQ, they record in their tables the address of neighbors, thereby establishing a reverse path. When the node which knows the path to destination or destination node itself receive RREQ, it send back Route Reply (RREP) packet to source node. This RREP packet is transmitted by using reverse path in formation in route table of each intermediate node. When the source node receives RREP packet, it can know the path to destination node and it stores the discovered path information in its route table. That is the end of route discovery 


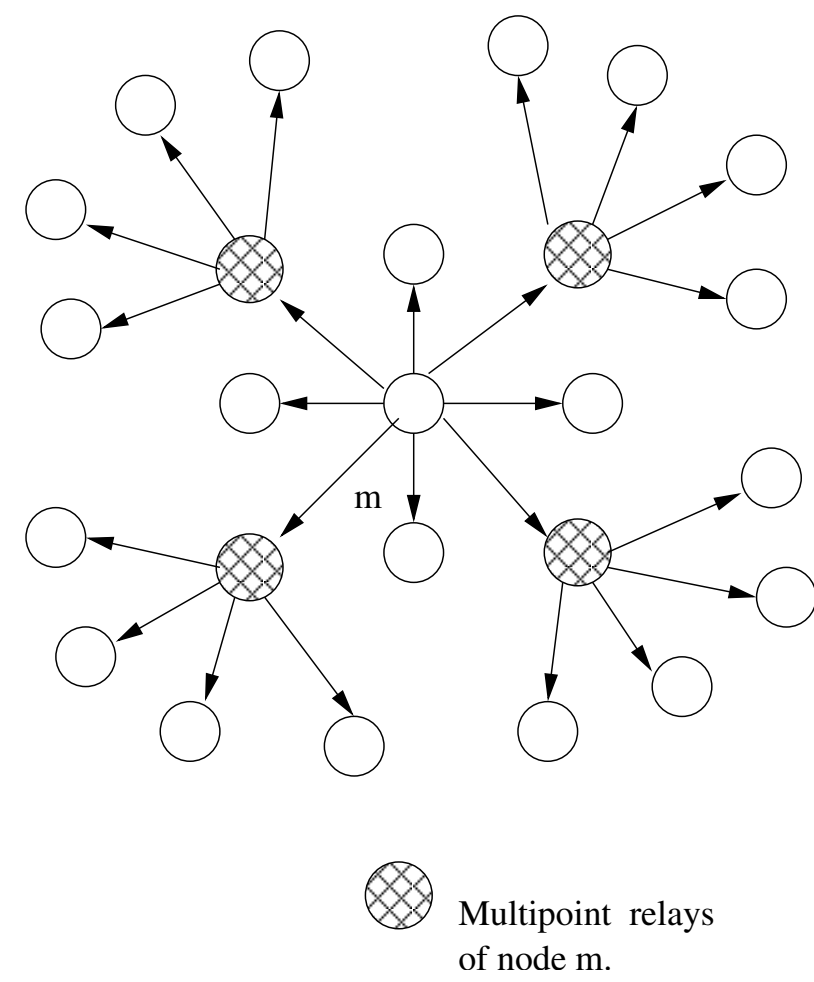

Figure 3. Multipoint relays of node $m$.

process. Then, AODV performs route maintenance process. In route maintenance process, each node periodically transmits a Hello message to detect link breakage [8].

\subsection{Topology}

For the physical layout of the WSN, two types of deployment has been studied so far: the random topology and lattice topology. In random topology, the nodes are supposed to be uniformly distributed, while in the lattice topology the nodes are vertexes of particular geometric shape, e.g. a square grid as depicted in Fig. 4. In this paper, we present the simulation results for both topologies $[9,10]$.

In lattice topology, in order to guarantee the connectedness of the network we should set the transmission range of every node to the step size, $d$, which is the minimum distance between two rows (or columns) of the grid. In fact, by this way the number of links that every node can establish (the node degree $\mathrm{D}$ ) is 4 . Nodes at the borders have $D=2$. The settings of our lattice are shown in Table1. The sensing range is assumed to be half of the transmission range.

In the case of random network, the setting of the transmission range is a little bit more complicated. In fact, since the position of nodes in the plane is a random variable, the number of neighboring links of a node is a random variable as well. First, we recall the following simple result
Table 1. Topology settings.

\begin{tabular}{|c|c|}
\hline \multicolumn{2}{|c|}{ Lattice } \\
\hline \hline Step $(\mathrm{m})$ & $d=\frac{L}{\sqrt{N}-1}$ \\
\hline Service Area Size $\left(\mathrm{m}^{2}\right)$ & $L^{2}=(800 \mathrm{x} 800)$ \\
\hline Number of Nodes & $N \in\{12,64\}$ \\
\hline Transmission Range $(\mathrm{m})$ & $r_{0}=d$ \\
\hline \hline \multicolumn{2}{|c|}{ Random } \\
\hline Density(nodes $\left./ \mathrm{m}^{2}\right)$ & $\rho \in\left\{25 \cdot 10^{-6}, 2 \cdot 10^{-4}\right\}$ \\
\hline Transmission Range $(\mathrm{m})$ & $r_{0}=180$ \\
\hline
\end{tabular}

borrowed from the random graph theory. Let $G(V, E)$ be the graph representation of the network, where $V$ is the set of vertexes and $E$ is the set of links. The network is said to be $k$-connected iff for every $(u, v) \in V$ there are $k$ disjoint paths connecting $u$ and $v$. The probability of $k$-connectivity is $P(1-$ conn $)$. Let suppose that nodes, or vertexes of $G(V, E)$ are uniformly distributed in the unitary Euclidean plane with intensity $\rho$. Asymptotically, the probability of connected network converges to 1 if the transmission range $r_{0}$ of every node is set as follows:

$$
r_{0} \geq \sqrt{\frac{\ln \left(\frac{\ln (P(1 \text {-conn })}{-\rho}\right)}{-\rho \pi}}
$$

where $P(1$-conn $)$ is the 1 -connectivity probability. The transmission range $r_{0}$ is the distance for which the received power is greater than a specific threshold. This threshold depends on the hardware, e.g. modulation and coding schemes, noise floor.

The network is supposed to have a single sink. In the lattice network, the $\mathrm{MN}$ is located at the top-right corner of the lattice. This situation is not far from the reality, because in some habitat monitoring applications, like the observation of mountains slope with landslide dangers, the sink could not be placed otherwise. However, in random networks, we let the MN to occupy any position in the plane.

The radio model parameters are listed in Table 2. The random topology is shown in Fig. 5. The sensor nodes are distributed in a random way in the field.

\subsection{Radio Model}

There are three basic models for the propagation of the radio signals of sensor nodes: Free space model, TwoRayGround model and Shadowing model [11, 12]. In a simple deterministic model, the received power $P_{r}$ at a certain distance $d$ is the same along all directions in the plane ${ }^{1}$. For

\footnotetext{
${ }^{1}$ We are considering $2 \mathrm{D}$ networks, but similar results hold also in the more general case of 3D networks.
} 
Table 2. Radio model and system parameters.

\begin{tabular}{|c|c|}
\hline \multicolumn{2}{|c|}{ Radio Model Parameters } \\
\hline Path Loss Coefficient & $\alpha=2.7$ \\
\hline Variance & $\sigma_{\mathrm{dB}}^{2}=16 \mathrm{~dB}$ \\
\hline Carrier Frequency & $916 \mathrm{MHz}$ \\
\hline Antenna & Omni \\
\hline Threshold (sensitivity) & $\gamma=-118 \mathrm{~dB}$ \\
\hline \multicolumn{2}{|c|}{ Other Parameters } \\
\hline Reporting Frequency & $\overline{T_{r}=[0.1,10000] \mathrm{pps}^{1}}$ \\
\hline Interface Queue Size & 50 packets \\
\hline UDP Packet Size & 100 bytes \\
\hline Detection Interval $\tau$ & $30 \mathrm{~s}$ \\
\hline
\end{tabular}

${ }^{1}$ packet per seconds

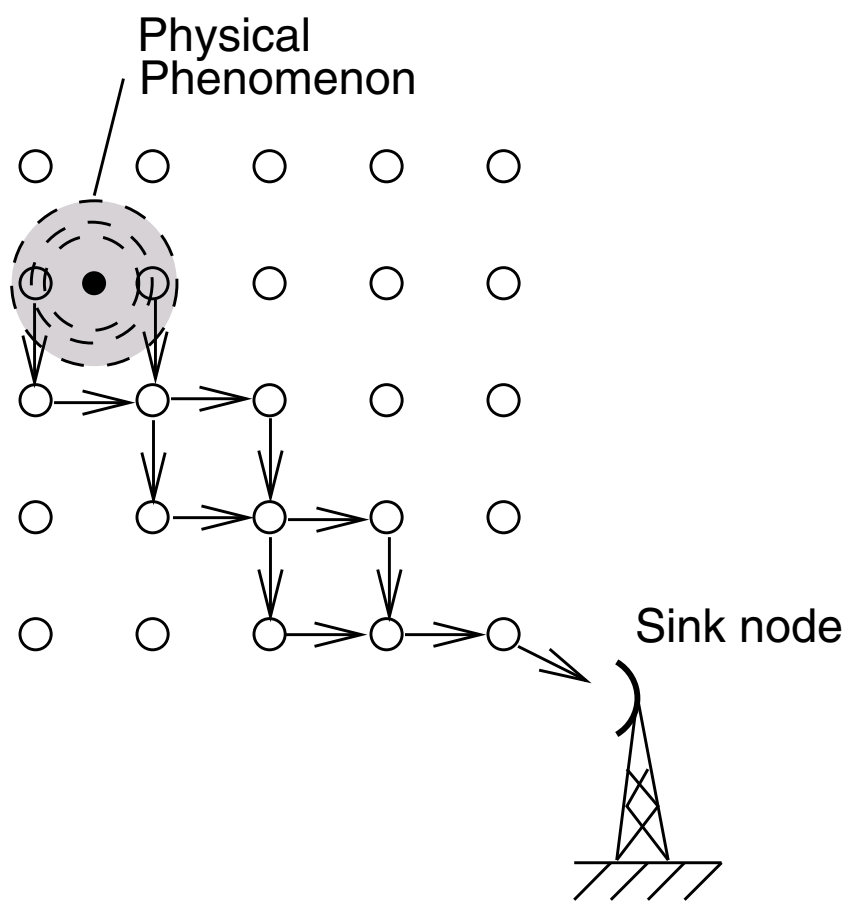

Figure 4. Lattice topology.

example, in case of Line Of Sight (LOS) propagation of the signal, the Friis formula predicts the received power as:

$$
\begin{gathered}
P_{r}(d)=P_{t}-\beta(\mathrm{dB}) \\
\beta=10 \log \left(\frac{(4 \pi d)^{2} L}{G_{t} G_{r} \lambda^{2}}\right)
\end{gathered}
$$

where $G_{r}$ and $G_{t}$ are the antenna gains of the receiver and the transmitter, respectively, $\lambda$ is the wavelength of the signal and $L$ the insertion loss caused by feeding circuitry of the antenna, and $\beta$ is the propagation pathloss. For omni-

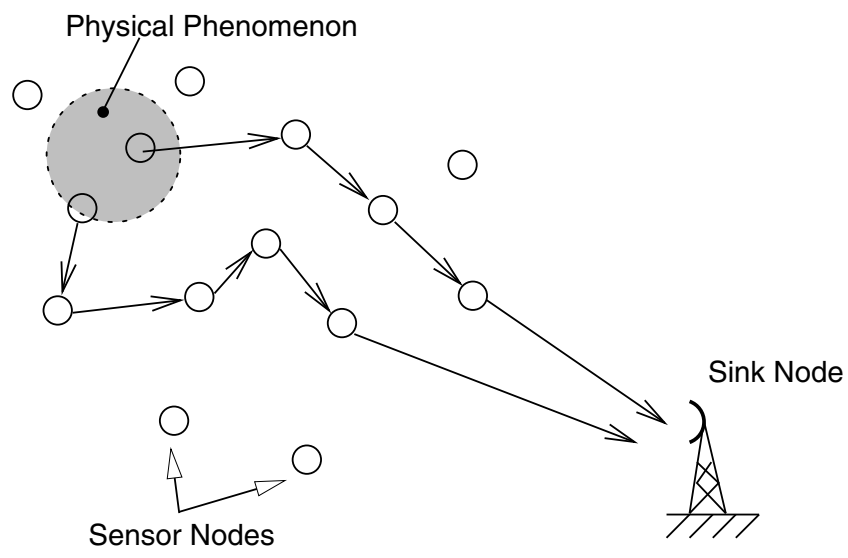

Figure 5. Random topology.

antennas, $G_{R}=G_{t}=1$. The signal decay is then proportional to $d^{2}$.

In our simulation system, we are using a more accurate model called TwoRayGround model, where in addition to the direct ray from the transmitter towards the receiver node, a ground reflected signal is supposed to be present. Accordingly, the received power now depends also on the antenna heights and the pathloss is:

$$
\beta=10 \log \left(\frac{(4 \pi d)^{4} L}{G_{t} G_{r} h_{t} h_{r} \lambda^{2}}\right)
$$

where $h_{r}$ and $h_{t}$ are the receiver and transmitter antenna heights, respectively. The power decreases faster than Eq. (2). The formula in Eq. (3) is valid for distances $d>d_{c}$, that is the distance far from the transmitting node [13].

\subsection{Event Detection and Transport}

Here, we use the data-centric model similar to [14], where the end-to-end reliability is transformed into a bounded signal distortion concept. In this model, after sensing an event, every sensor node sends sensed data towards the MN. The transport used is a UDP-like transport, i.e. there is not any guarantee on the delivery of the data. While this approach reduces the complexity of the transport protocol and well fit the energy and computational constraints of sensor nodes, the event-reliability can be guaranteed to some extent because of the spatial redundancy. The sensor node transmits data packets reporting the details of the detected event at a certain transmission rate $^{2}$. The setting of this parameter, $T_{r}$, depends on several factors, as the quantization step of sensors, the type of phenomenon, and the desired level of distortion perceived at the MN. In [14], the

${ }^{2}$ Note that in the case of discrete event, this scheme is a simple packet repetition scheme. 


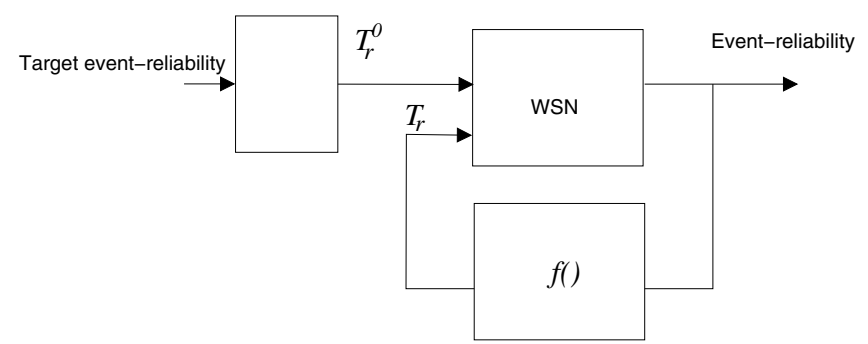

Figure 6. Representation of the transport based on the event-reliability.

authors used this $T_{r}$ as a control parameter of the overall system. For example, if we refer to event-reliability as the minimum number of packets required at $\mathrm{MN}$ in order to reliably detect the event, then whenever the MN receives a number of packets less than the event-reliability, it can instruct sensor nodes to use a higher $T_{r}$. This instruction is piggy-backed in dedicated packets from the MN. This system can be considered as a control system, as shown in Fig. 6, with the target event-reliability as input variable and the actual event-reliability as output parameter. The target event-reliability is transformed into an initial $T_{r}^{0}$. The control loop has the output event-reliability as input, and on the basis of a particular non-linear function $f(\cdot), T_{r}$ is accordingly changed. We do not implement the entire control system, but only a simplified version of it. For instance, we vary $T_{r}$ and observe the behavior of the system in terms of the mean number of received packets. In other words, we open the control loop and analyze the forward chain only.

\section{Goodput and Depletion}

In this section, we introduce the concept of Goodput and Depletion. We consider that after a sensor node detects the physical phenomenon, it sends the packets to the sink node via a routing protocol. The ability for transmitting packets for different protocols is different. Also, the Goodput of a protocol is affected by many network parameters such as wireless transmission radio model, network topology, and transmission frequency [4]. In order to compare the performance of different protocols, we consider the same simulation environment. We use for evaluation Goodput, which is the maximum of the event-reliability. The Goodput is defined at the sink, and it is the received packet rate divided by the sent packets rate. Thus:

$$
G(\tau)=\frac{N_{r}(\tau)}{N_{s}(\tau)}
$$

where $N_{r}(\tau)$ is the number of received packet at the sink, and the $N_{s}(\tau)$ is the number of packets sent by sensor nodes which detected the phenomenon. Note that the eventreliability is defined as $G_{R}=\frac{N_{r}(\tau)}{R(\tau)}$, where $R$ is the required number of packets or data in a time interval of $\tau$ seconds.

As long as the WSN is being used, a certain among of energy will be consumed. The energy consumption rate diretly affects the life-time of the network, i.e. the time after which the WSN is unusable. According, we define the energy consumed by the network in the detection interval $\tau$ as:

Energy consumed $\triangleq \frac{\text { Ini Tot. Energy }- \text { Fin Tot. Energy }}{\tau}$.

However, in order to compare the performances of the scaled networks, it is better to define the mean energy Depletion rate per node as:

$$
\Delta(\tau) \triangleq \frac{E_{I}-\bar{e}(\tau)}{\tau}=\frac{N E_{I}-\sum_{i=1}^{N} e_{i}(\tau)}{N \tau}
$$

where $e_{i}(t)$ is the node energy at time $t$ and the means are computed over the number of nodes. The number of nodes $N$ is set as power of integers in order to analyse the behaviour of the scaled versions of the network.

\section{Simulation Results}

In this section, we present the simulation results. We implemented the simulation system using ns-2 simulator, with the support of NRL libraries [15]. The initial position of the phenomenon node is varied along the simulation runs. The sample averages of Eq. (4) and Eq. (5) are computed over 20 simulation runs and are plotted in Fig. 7 and Fig. 8, respectively, with respect to the particular radio model used.

In Fig. 7 is shown the average value of Goodput using AODV and OLSR protocols. The Goodput is an decreasing function of $T_{r}$. As $T_{r}$ increases, the number of sent packet by sensing node is lower than the number of packets used by routing protocol. In case of AODV, the Goodput decreases with the sensor nodes increasing. It should be noted that when the number of sensor nodes is increased, the number of routes have increased, thus the searching time to find a route also is increased. In case of OLSR, the Goodput is lower than AODV when the transmission rate is lower than 10pps. But, the transmisson rate of OLSR is better than AODV when the transmission rate is larger than 10pps. This is because, when the transmission rate is less than 10pps, all sensor nodes use flooding in order to establish the MPR. After, the MPR are selected, the Goodput of OLSR becomes better than AODV because the packets are transmited with a high success rate to the sink. This shows that OLSR protocol can be used in large scale sensor networks. 
In Fig. 8 is shown the average value of Depletion for AODV and OLSR protocols. When the transmisson rate is larger than $1 \mathrm{pps}$, the Depletion of OLSR is better than AODV. This is because after establishing MPR nodes, the number of relay nodes is decreased.

In following, we explain the results of Goodput for OLSR protocol with defferent topologies. In Fig. 9 is shown the Goodput for regular and random topologies. In both cases, when the number of nodes is increased, the Goodput is increased. In case of random topology, the Goodput is better than the case of regular topology. In Fig. 10 is shown the Depletion for regular and random topologies. In case of regular topology, the Depletion is better than the case of random topology .

\section{Conclusions}

In this paper, we presented the implementation of a simulation system for WSNs using ns-2. We considered for simulations OLSR and AODV protocols. From the simulation results, we conclude as follows.

- In case of AODV, the Goodput decreases with the increase of number of sensor nodes.

- In case of OLSR, the Goodput is increased when the number of nodes is increased.

- The Goodput of AODV is better than OLSR when the transmission rate is lower 10pps, but when the transmission rate is larger than 10pps the Goodput of OLSR is better than AODV.

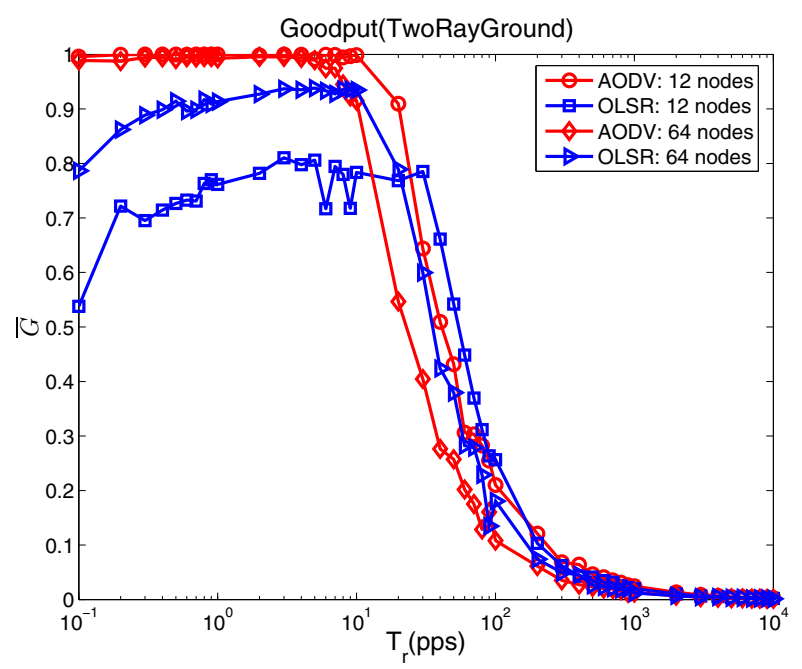

Figure 7. Goodput for OLSR and AODV.

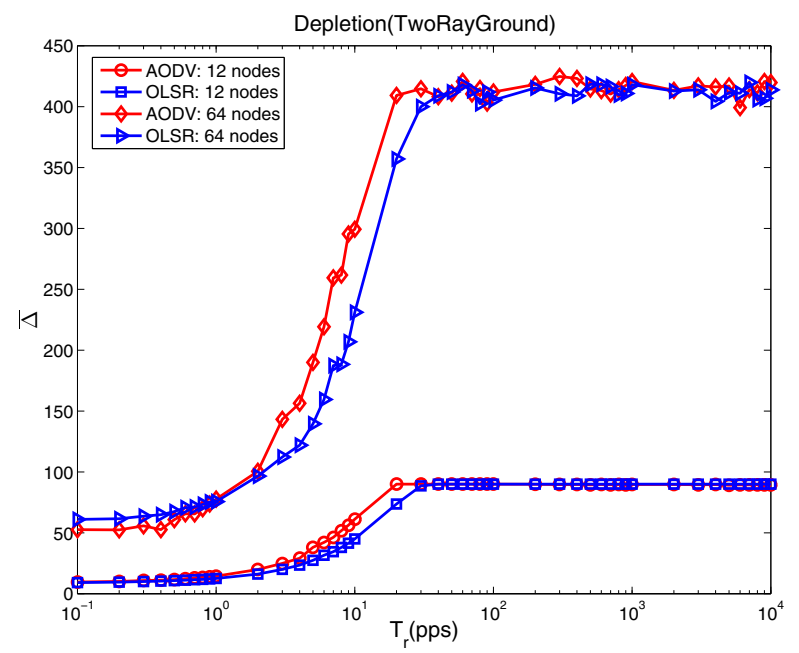

Figure 8. Depletion for OLSR and AODV.

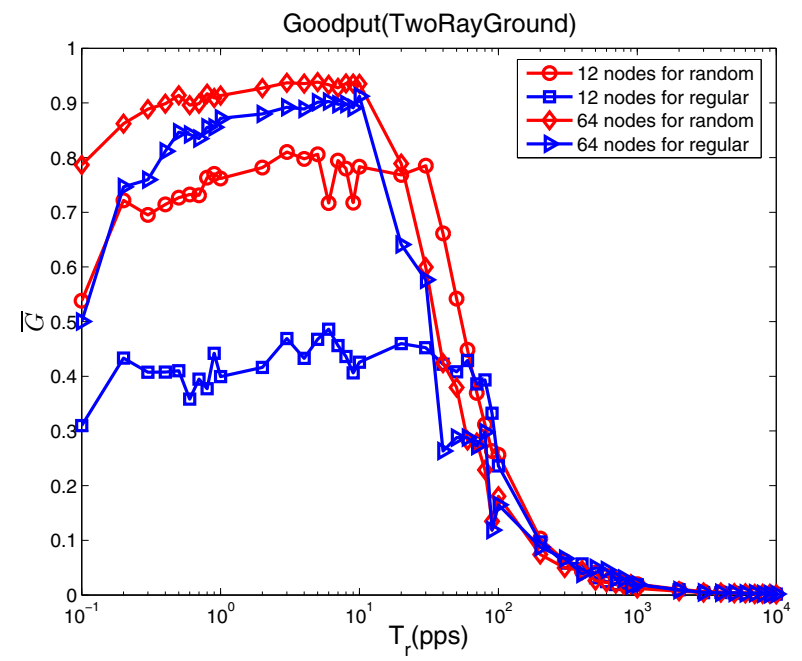

Figure 9. Goodput for different topologies (OLSR).

- Comparing the Depletion of AODV and OLSR, we see that when the transmission rate is lower $1 \mathrm{pps}$, the Deplation of AODV is better than OLSR. But, when the transmission rate is larger than 10pps, the Depletion of OLSR is better than AODV.

- Comparing the Goodput and Depletion for different topologies, both the Goodput and Depletion of OLSR in the case of random topology are better than in case of regular topology.

In the future, we would like to carry out more extensive simulations for a large scale sensor network and other proto- 


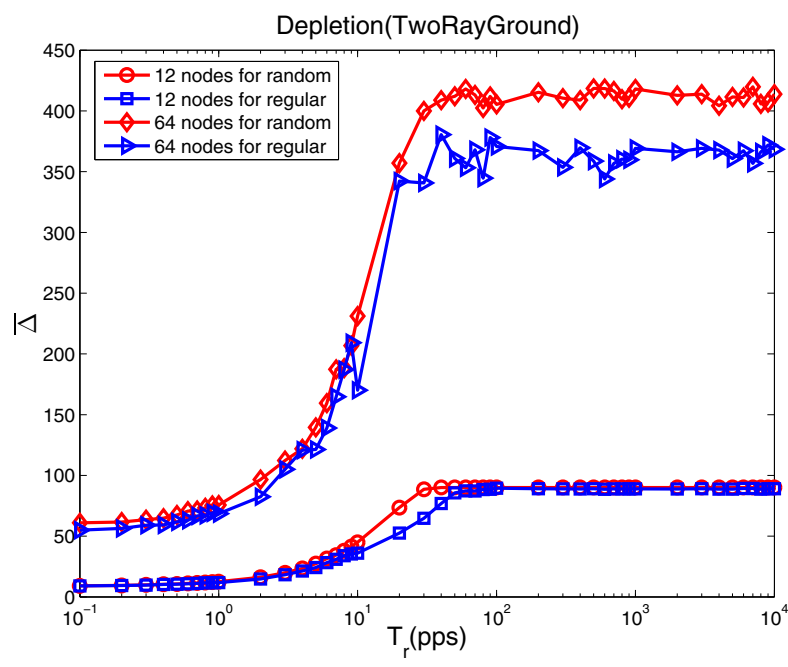

Figure 10. Depletion for different topologies (OLSR).

cols. We also would like to consider the case of Shadowing radio model.

\section{Acknowledgment}

The authors would like to thank International Communications Foundation (ICF) of Japan and Japanese Society for the Promotion of Science (JSPS) for supporting this work.

\section{References}

[1] S. Giordano and C. Rosenberg, "Topics in Ad Hoc and Sensor Networks", IEEE Communication Magazine, Vol. 44, No. 4, pp. 97-97, 2006.

[2] J. N. Al-Karaki and A. E. Kamal, "Routing Techniques in Wireless Sensor Networks: A Survey", IEEE Wireless Communication, Vol. 11, No. 6, pp. 6-28, December 2004.

[3] G. W.-Allen, K. Lorincz, O. Marcillo, J. Johnson, M. Ruiz, J. Lees, "Deploying a Wireless Sensor Network on an Active Volcano", IEEE Internet Computing, Vol. 10, No. 2, pp. 1825, March, 2006.

[4] O. Younis, S. Fahmy, "HEED: A Hybrid, Energy-efficient, Distributed Clustering Approach for Ad-hoc Sensor Networks", IEEE Transactions on Mobile Computing, Vol. 3, No. 4, pp. 366-379, 2004.

[5] T. Yang, G. De Marco, M. Ikeda, L. Barolli, "A Case Study of Event Detection in Lattice Wireless Sensor Network with Shadowing-Induced Radio Irregularities", Proc. of MoMM2006, Yogyakarta, Indonesia, pp. 241-250, December 2006.
[6] T. Clausen and P. Jacquet (editors). "Optimized Link State Routing Protocol (OLSR)”, RFC 3626, IETF Network Working Group, October 2003.

[7] A. Qayyum, L. Viennot, A. Laouiti, "Multipoint Relaying: An Efficient Technique for flooding in Mobile Wireless Networks," Tech. Rep. RR-3898, INRIA, http://www.inria.fr, February 2000.

[8] Charles E. Perkins and Elizabeth M. Royer. "Ad hoc OnDemand Distance Vector Routing. In Proceedings of the 2nd IEEE workshop on Mobile Computing Systems and Applications (WMCSA'99), pages 90-100, February 1999. New Orleans, LA.

[9] T.S. Rappaport, "Wireless Communications", Prentice Hall PTR, 2001.

[10] C. Cooper. A note on the connectivity of 2-regular digraphs. Random Structures Algorithms, 4:469-472, 1993.

[11] W. Ye, J. Heidemann, D. Estrin, Medium Access Control with Coordinated Adaptive Sleeping for Wireless Sensor Networks", IEEE/ACM Transaction Networking, Vol. 12, No. 3, pp. 493-506, 2004.

[12] G. Zhou, T. He, S. Krishnamurthy, J. A. Stankovic, ”Models and Solutions for Radio Irregularity in Wireless Sensor Networks", ACM Transaction on Sensors Network, Vol. 2, No. 2, pp. 221-262, 2006.

[13] M. Zuniga and B. Krishnamachari, "An Analysis of Unreliability and Asymmetry in Low-power Wireless Links", http://www-scf.usc.edu/marcozun/, 2006.

[14] Özgür B. Akan and I. F. Akyildiz. Event-to-sink reliable transport in wireless sensor networks. IEEE/ACM Transactions on Networking, 13(5):1003-1016, 2005.

[15] I. Donward, "NRL's Sensor Network Extension to NS-2", http://pf.itd.nrl.navy.mil/nrlsensorsim/, 2004. 